\title{
Tamper Evident Metal Container Closure
}

National Cancer Institute

\section{Source}

National Cancer Institute. Tamper Evident Metal Container Closure. NCI Thesaurus. Code C96117.

A closure/finish of a closure/container system designed to make it difficult to achieve the first removal of a closure from a container without it being detectable by subsequent users that the package seal has been breached (e.g., aluminum overseal). 